\section{About the ways of discovery}

Daniil Makarov, a post-graduate student

Psychological Institute of the Russian Academy of Education

Discovery of new knowledge is a main factor of creativity and progress of human culture. However, explanation concerning prerequisites of person's ability to discover new knowledge still remains a challenge for most of international psychological communities. In this review, we compare two theories proposing their answers to this question, which are the theory of resolution of invention-related tasks (TIPS) by G. Altshuller, and the theory of creation by $D$. Bogoyavlenskaya.

\section{T.I.P.S. and L.S.C.P. by G. Altshuller}

G. Altshuller states that there are phenomena discoveries (detection) and explanation discoveries (theories). He proposed that theories are harder to discover, therefore they are a more rare type of discoveries than detections.

From 1946 to 1971, G. Altshuller examined 40,000 patents, classified the solutions that he found in five levels. He detected general algorithms and forty standard methods that were used by the inventors.

However, TIPS apologists admit that if one learns TIPS, he or she does not necessarily become a creative person.

G. Altshuller and I. Vertkin examined more than a thousand of creative people's biographies and came to the conclusion that life of creative people may be envisaged as a path of creative purpose for which they live trying to avoid any hindrances.

Based on this research, the authors determined a set of creative person traits: 1. Altshulle G.S., Vertkin, I.M. 1994: How to become a Genius: the
Life
1. Socially useful purpose

2. A set of plans to achieve the purpose

3. High performance

4. Good problem solving technique

5. Ability to defend one's ideas

6. Productivity

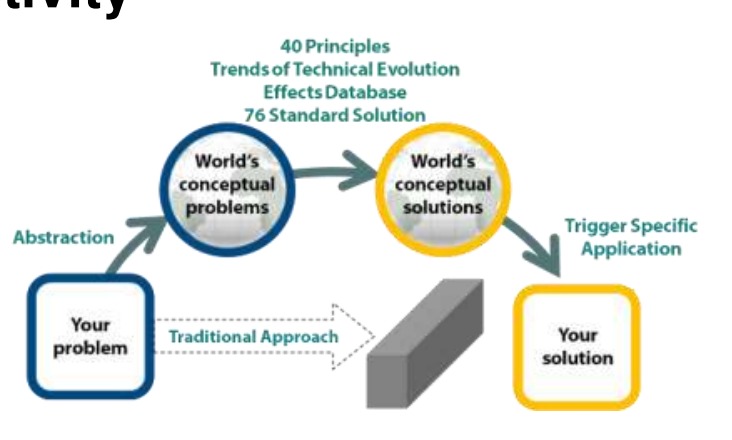

Theory of creativity by D.

Bogoyavlenskaya

The "Creative Field" method, which is developed within the framework of D.B. Bogoyavlenskaya theory, allows one not only to diagnose creative abilities but also to predict the possibilities of innovative achievements.

The method "Creative Field" is based on providing subjects with the same type of tasks in an unusual field, which makes it possible for the subjects to discover patterns on their own initiative.

Creation of new algorithms for solving problems comes as a result of finding ways to meet a cognitive need. This idea is also valid for "goals", "long-term plans", developed "skills" and "habits", as well as for any other methods of organizing life.

A person directed by a dominance of a cognitive need can and will most likely use such methods of organizing his or her life, but this is not what determines his or her inclination.

\section{Levels of intellectual activity: \\ - Stimulated = Heuristic = Creative}

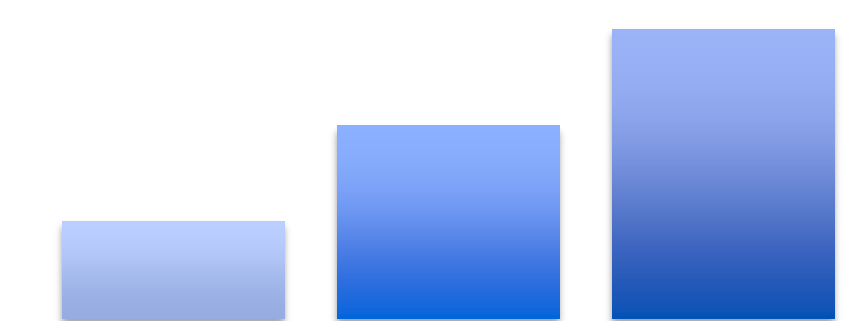

What is a way of discovery?

Many thinkers and researchers explained making a discovery as a result of using techniques of creativity unlocking. The proo of it is such methods as brainstorming, lateral thinking etc.

However, the majority of people who made breakthrough discoveries did not know about TIPS or other methods of creativity unlocking Everything that one can imitate and lie about (socially useful purpose, productivity) canno be considered as a proof of creativity or predictor of discovery.

If we are interested in psychological predictors of making a discovery, we have to focus on:

1. The level of mastering an activity. To develop an activity, one has to first master it. 2. The levels of intelligence development. To be able to master an activity, one has to have enough of general intellectual abilities for that.

3. The place of cognitive motivation in the motivational structure of personality. To want to explore an activity beyond situationa requirements, a person has to aspire to cognition first, it has to be person's strongest aspiration.

\section{Results and Conclusions}

In D. Bogoyavlenskaya theory, a specific psychological explanation of making a discovery is given.

If a cognitive need dominates in a personality structure, it leads to making discoveries. And despite the fact that the scientist does not directly say that there is a greater cognitive motivation behind explanations than behind detections, this idea is clearly between the lines.

Making a discovery becomes possible when an activity is successfully mastered, but interest in it remains.

Making a discovery is the result of human activity that is no longer a response to stimuli.

Mechanism of making a discovery is the same for every cultural domain.

Making a discovery accompanies decentralization of one's personality, person's concentration on a process of an activity and anticipation of the joy of grasping the knowledge.

The desire to bring benefits can support creative orientation of an individual, but is not the main determining factor for it.

Personal development and creativity are correlated if we understand personal development as selfless passion about commitment to a certain activity. 